\title{
Multiple Dose Pharmacokinetics of Paroxetine in Children and Adolescents with Major Depressive Disorder or Obsessive-Compulsive Disorder
}

\author{
Robert L Findling*,', Gianluca Nucci ${ }^{2}$, Antoni A Piergies ${ }^{3}$, Roberto Gomeni ${ }^{2}$, Edward I Bartolic ${ }^{4}$, \\ Regan Fong ${ }^{5}$, David J Carpenter ${ }^{5}$, J Steven Leeder ${ }^{6}$, Andrea Gaedigk ${ }^{6}$ and Theodore M Danoff ${ }^{5}$ \\ 'Departments of Psychiatry and Pediatrics, Case Western Reserve University, University Hospitals of Cleveland, OH, USA; \\ ${ }^{2}$ GlaxoSmithKline,Verona, Italy; ${ }^{3}$ PAREXEL, Baltimore, MD, USA; ${ }^{4} 3$ Research, Basking Ridge, NJ, USA; ${ }^{5}$ GlaxoSmithKline, King of Prussia, \\ PA, USA; 'Division of Pediatric Pharmacology and Medical Toxicology, Children's Mercy Hospital and Clinics, Kansas City, MO, USA
}

\begin{abstract}
The current study examined the pharmacokinetics (PK), safety, and tolerability of paroxetine after repeated multiple oral dosing in children and adolescents with major depressive or obsessive-compulsive disorder. In this 6-week, open-label, repeat dose, dose-rising study, 62 patients (27 children and 35 adolescents) were treated with paroxetine $10 \mathrm{mg} /$ day for the first 2 weeks of the study, $20 \mathrm{mg} /$ day for the next 2 weeks, and $30 \mathrm{mg} /$ day for the final 2 weeks. Pharmacokinetic sampling and safety assessments occurred at baseline and subsequently on the final treatment day of each dosing level. Between-patient variability in PK was pronounced at the $10 \mathrm{mg}$ dose level, but markedly reduced at higher doses. A supra-proportional increase in plasma concentrations with increasing dose was evident in both age groups. Data for $C_{\max }$ and $A \cup C_{(0-24)}$ indicated that, at each dose level, paroxetine steady-state systemic exposure was higher in children than in adolescents. The differences between age groups, however, diminished with each increasing dose, and were virtually abolished when differences in weight among different age groups were considered. Stepwise regression analysis indicated that both oral clearance and volume of distribution were highly dependent on paroxetine dose, cytochrome P4502D6 genotype, and weight ( $p<0.000 \mathrm{I}$ ), but not age or sex. Paroxetine was generally safe and well tolerated in both age groups, with the most frequently observed adverse events being largely consistent with those observed in prior paroxetine studies of adult psychiatric patients. Certain gastrointestinal and behavioral activation events (aggressive reaction and nervousness) were reported more frequently in the youngest age group. Neuropsychopharmacology (2006) 3 I, I274- 1285. doi:I 0.1038/sj.npp. I300960; published online 23 November 2005
\end{abstract}

Keywords: paroxetine; pharmacokinetics; children; adolescents; depression; obsessive-compulsive disorder

\section{INTRODUCTION}

Paroxetine hydrochloride $\left(\right.$ Paxil $^{\circledR}$, Seroxat ${ }^{\circledR}$ ) is a serotonin selective re-uptake inhibitor (SSRI) (Beique et al, 1998; Thomas et al, 1987), and is the hydrochloride salt of a phenylpiperidine (Tulloch and Johnson, 1992). In adults, paroxetine has been shown to be superior to placebo in the treatment of major depressive disorder (MDD), obsessive-compulsive disorder (OCD), and several other anxiety disorders (Wagstaff et al, 2002). While results from studies without placebo control have described beneficial effects of paroxetine for juvenile MDD (Braconnier et al, 2003; Findling et al, 2002; Rey-Sanchez and GutierrezCasares, 1997), evidence from randomized, placebo-con-

\footnotetext{
*Correspondence: Dr RL Findling, Department of Psychiatry, Case Western Reserve University, University Hospitals of Cleveland, I I 100 Euclid Avenue, Cleveland, OH 44106-5080, USA, Tel: + I 216844 | 707, Fax: + | 216844 5883, E-mail: robert.findling@uhhs.com Received I June 2005; revised 2 September 2005; accepted 23 September 2005

Online publication: I I October 2005 at http://www.acnp.org/citations/ Npp I 0 I I05050356/default.pdf
}

trolled clinical trials has been equivocal. These trials have failed to demonstrate statistical separation from placebo on primary outcome measures, but have suggested efficacy on some secondary measures (Keller et al, 2001; Milin et al, 1999). In contrast, there is stronger evidence that paroxetine may be an effective treatment for young people suffering from OCD (Diler et al, 2000; Geller et al, 2003, 2004).

Paroxetine's modulation of serotonergic neural transmission is thought to be primarily responsible for its therapeutic properties (DeVane, 1992). It appears that paroxetine has a similar mechanism of action in children, adolescents, and adults (Findling et al, 2002; Lund et al, 1979; Marsden et al, 1987).

Paroxetine is initially metabolized through demethylation (Haddock et al, 1989). This removal of the carbon at the methylenedioxy position is catalyzed by cytochrome P4502D6 (CYP2D6) (Bloomer et al, 1992; Crewe et al, 1992), yielding a catechol that is subsequently both methylated and conjugated (Tulloch and Johnson, 1992). The metabolites of paroxetine are considered to be pharmacologically inactive (Kaye et al, 1989). 
The first report to specifically examine the biodisposition of paroxetine in youths described paroxetine's first-dose pharmacokinetics (PK), multiple-dose concentrations, and the effects of CYP2D6 phenotype on the compound's disposition. This work was conducted in a group of children and adolescents with MDD (Findling et al, 1999). For first-dose $\mathrm{PK}$, the authors found that children and adolescents had values similar to adults (Kaye et al, 1989) for time to maximum concentration $\left(T_{\max }\right)$, with a mean value of $5.7 \mathrm{~h}$. The investigators noted that, as in adults, there were large interindividual differences in paroxetine PK parameters. In addition, the authors reported that only a modest fraction of paroxetine was excreted unchanged in the urine of pediatric patients, which is a similar finding to the adult population (Lund et al, 1979). Conversely, the authors found that the average half-life $\left(t_{1 / 2}\right)$ of a first dose of paroxetine was substantially shorter than what had been previously described in adults $\left(t_{1 / 2}=11.1 \mathrm{~h}\right.$ in pediatrics $v s$ more than $20 \mathrm{~h}$ in adult studies) (Kaye et al, 1989).

With regard to multiple-dose paroxetine concentrations in pediatric patients, Findling et al (1999) found two key similarities in the PK of paroxetine between pediatric patients and adults (Kaye et al, 1989; Lund et al, 1979; Raptopoulos et al, 1989; Sindrup et al, 1992a). First, nonlinear PK were observed. Also, modest intraindividual variability in plasma drug concentrations was found by the end of 1 week of paroxetine therapy. In addition, it was shown that activity with respect to CYP2D6 phenotype could affect the exposure of paroxetine in children and adolescents (Findling et al, 1999). These phenomena have also been reported in adults (Ozdemir et al, 1999; Sindrup et al, 1992b) and are due to the involvement of the saturable enzyme CYP2D6 in paroxetine clearance.

As there are limited data regarding the multiple-dose PK parameters of paroxetine in pediatric patients, this study was undertaken to further describe the paroxetine PK after repeated multiple oral dosing. Another purpose of this study was to examine the safety and tolerability of paroxetine following repeat oral doses to children and adolescents.

\section{METHODS}

\section{Subjects}

Medically healthy children (ages 7-11 years) and adolescents (ages 12-17 years) meeting the Diagnostic and Statistical Manual of Mental Disorders, Fourth Edition (DSM-IV) diagnostic criteria for a primary diagnosis of MDD or OCD (American Psychiatric Association, 1994), who were judged to likely be adherent with study procedures, were eligible to participate. Diagnoses were made based on the results of the Schedule for Affective Disorders and Schizophrenia for School-Age Children-Present and Lifetime Version (KSADS-PL) (Kaufman et al, 1997). In order to participate in this trial, patients also needed a Children's Depression Rating Scale-Revised (CDRS-R) (Poznanski et al, 1985) score $\geqslant 45$ or a Children's Yale-Brown Obsessive-Compulsive Scale (CY-BOCS) (Scahill et al, 1997) score $\geqslant 16$, both of which are indicative of at least moderate symptom severity.

Key exclusion criteria included a clinically relevant medical history or significant abnormality on screening medical assessments, positive drug screen, current suicidal or homicidal risk as judged by the investigator, a clinically predominant Axis I disorder other than MDD or OCD, and a history of nonresponse or hypersensitivity to SSRI treatment. In addition, the use of fluoxetine, depot antipsychotics, or nonprescription drugs that might affect cytochrome P450 enzymes within 3 months prior to starting study medication was exclusionary. Pregnant or nursing females and females of childbearing potential who were not willing or able to use an acceptable means of birth control throughout the study were also excluded.

\section{Study Design}

This 6-week, multi-center, open-label, repeat dose, doserising study in children and adolescents with either MDD or OCD was conducted at 12 clinical research units (CRUs) in the United States. Each participating institution's Institutional Review Board for Human Investigation approved the procedures of this study. The patients' guardians provided written informed consent and patients gave written informed assent prior to the initiation of any study procedures.

Within 30 days prior to the first dose of study medication being administered, patients visited the CRU for screening assessments. Patients underwent psychiatric and physical evaluations and completed psychometric measures (see below). Patients meeting all study entry criteria were eligible to enter the 6-week, open-label, dose-rising stage of the study. Patients were treated with paroxetine $10 \mathrm{mg} /$ day for the first 2 weeks of the study (days 1-14), paroxetine $20 \mathrm{mg} /$ day for the next 2 weeks (days 15-28), and then paroxetine $30 \mathrm{mg} /$ day for 2 more weeks (days 29-42). Throughout the course of the trial, paroxetine was given in the morning as a single daily dose.

Patients and their families had four scheduled visits to the CRU during the dose-rising period. There was a baseline visit on the first dosing day, and then visits occurred every other week thereafter, on the final day of each dosing level (ie days 14, 28, and 42), for PK sampling and safety assessments. Between visits to the CRU, patients were followed on an outpatient basis via telephone contacts by study personnel every 3 to 4 days. During each telephone contact, adverse events (AEs) and concomitant medication use were assessed. Study personnel supervised paroxetine administration on study visit days. Otherwise, drug adherence was assessed with dosing diaries and tablet counts. The use of concomitant or prohibited medications was assessed by direct query at each study visit.

Patients were precluded from consuming ethanol, taking most other prescription drugs, nonprescription drugs, illicit drugs, or herbal/naturopathic preparations throughout the trial. Topical agents, inhaled steroids, some analgesics/ antipyretics, and antibiotics that would not interfere with data interpretation were allowed. As grapefruit or grapefruit juice might affect drug biodisposition (Bailey et al, 1998), consumption of grapefruit or grapefruit juice was precluded within 7 days of receiving the first dose of paroxetine and throughout the trial. Consumption of drinks and foods containing caffeine and other xanthines was prohibited $24 \mathrm{~h}$ prior to and during PK sampling.

After the 6-week dose-rising stage of the study was completed, patients could either enter a separate open-label 
extension study or a 2-week, dose-tapering procedure, whereby the daily dose of paroxetine was decreased by $10 \mathrm{mg}$ per week, until the daily dose was $10 \mathrm{mg}$ for 1 week prior to discontinuing study participation.

\section{Pharmacokinetic Sampling Strategy}

PK sampling for measurement of plasma paroxetine concentrations was conducted over, approximately, a 24-h period at the CRU following the final dose at each dosing level in the dose rising portion of the trial (days 14, 28, and 42). Some flexibility was permitted in the duration of the dosing periods. However, to ensure that steady-state conditions had been established, PK measurements were only made after at least 11 days of dosing at each dose level. Samples were obtained predose (no more than 15 min prior to dosing), and at $1,2,3,5,8,12( \pm 1)$, and $24( \pm 2) \mathrm{h}$ postdose. Dosing of paroxetine on these days occurred $15 \mathrm{~min}$ after the patients had completed breakfast. Patients received meals $4,9-10$, and $24 \mathrm{~h}$ postdose. Water was allowed ad libitum starting $2 \mathrm{~h}$ postdose, and caffeine-free soft drinks and fruit juices (except grapefruit juice) were allowed ad libitum beginning $4 \mathrm{~h}$ after dosing. Tobacco use was not permitted during the sampling period. An intravenous cannula, kept patent with a heparin solution (100 units $/ \mathrm{ml}$ ), with a topical anesthetic preparation used for cannula insertion, was permitted in order to reduce patient discomfort.

Each sample for PK analysis consisted of a $1.5 \mathrm{ml}$ sample of blood that was drawn into EDTA-coated tubes. The blood samples were placed temporarily on water-ice and then centrifuged within $1 \mathrm{~h}$ of collection to separate the plasma. Plasma was then transferred to polypropylene tubes and frozen immediately at approximately $-20^{\circ} \mathrm{C}$ until shipment to a central laboratory for quantitative analysis. Samples remained frozen until the analysis was performed.

\section{Safety Assessments}

A complete psychiatric history and medical history (including medication history for at least 3 months prior to enrollment) was obtained during the screening period. In addition, a physical examination (including height and weight measurements as well as a resting, sitting blood pressure and pulse) and a 12-lead electrocardiogram (ECG) were performed prior to medication dosing. A blood specimen was obtained for a hematology profile, chemistry profile, and thyroid profile, along with a urine specimen for urinalysis and urine drug screen. All female patients had a serum pregnancy test.

At baseline, medical and medication histories were updated, a limited physical examination was performed, vital signs were repeated, blood and urine specimens were obtained for clinical laboratory tests, and females had urine and serum samples obtained for pregnancy tests. A negative result on the urine pregnancy test was required prior to receiving the first dose of study medication.

A limited physical examination and height and weight measurements were also obtained prior to dosing on days 14, 28, and 42. Resting, sitting blood pressure, and pulse were obtained prior to dosing and $24 \mathrm{~h}$ postdose on days 14 ,
28, and 42. A 12-lead ECG, urine, and blood samples for laboratory tests, and a serum pregnancy test (females only) were obtained on day 42.

Patients were queried about the presence of physical and psychiatric symptoms at baseline, prior to initiating paroxetine therapy. During visits to the CRU on days 14, 28 , and 42 , AEs were assessed by nonleading queries (eg 'How do you/your child feel?') and by nursing and physician observation prior to dosing and at 13 and $24 \mathrm{~h}$ postdose. AEs occurring between visits were also assessed by nonleading queries at each study visit and each of the telephone contacts between study visits.

\section{Psychometric Assessments}

The K-SADS-PL, along with the CY-BOCS or CDRS-R (depending upon the patient's primary diagnosis, ie OCD or MDD), was administered during the screening period. A Clinical Global Impression (National Institute of Mental Health, 1985) Severity of Illness (CGI-S) rating was made at the screening visit. In addition, ratings with the CGI-S and Global Improvement scale (CGI-I) rating were made on day 42. However, no formal analysis was planned or performed with the CGI-S and CGI-I data.

\section{CYP2D6 Genotyping}

A separate blood sample was obtained $12 \mathrm{~h}$ postdose on day 14 for CYP2D6 genotyping. Additional consent/assent procedures were required for the collection of this sample. However, overall study enrollment was not predicated on agreement by the patient and/or parent/guardian to provide the genotyping sample.

Genomic DNA was isolated using the QIAamp DNA Blood Mini Kit (Qiagen, Valencia, CA), and CYP2D6 genotyping was conducted for $C Y P 2 D 6^{\star} 2,{ }^{\star} 3,{ }^{\star} 4,{ }^{\star} 5,{ }^{\star} 6$, ${ }^{\star} 7,{ }^{\star} 8,{ }^{\star} 9,{ }^{\star} 10,{ }^{\star} 11,{ }^{\star} 12,{ }^{\star} 15,{ }^{*} 17,{ }^{\star} 29,{ }^{*} 35,{ }^{\star} 36,{ }^{\star} 40$, ${ }^{*} 41$ [2988G], ${ }^{*} 41$ [2988A], ${ }^{*} 42$, and $\star^{*} 45 / 46$ alleles and the presence of ${ }^{\star} 1 \times 2,{ }^{\star} 2 \times 2$, and ${ }^{\star} 10 \times 2$ gene duplications, as originally described (Gaedigk et al, 1999) and subsequently modified (Gaedigk et al, 2002, 2003a, b, 2005a, b). Of note, personnel involved in the collection and evaluation of safety data were blinded to the results of CYP2D6 genotyping until all safety data were collected and all safety data queries were resolved.

To simplify interpretation of genotype data and to help assess the expected pharmacokinetic consequence of a given genotype, the analyses took advantage of the 'activity score' concept recently introduced for CYP2D6 (Zineh et al, 2004). A value indicating the relative activity of each CYP2D6 allele, based on a score of ' 1 ' for the fully functional reference $C Y P 2 D 6^{\star} 1$ allele, was assigned to each allele as follows:

Value assigned
2
1
0.75
0.5
0


The final activity score was based on the sum of the individual values as determined by the genotyping results for each subject. Subjects categorized as extensive metabolizers (activity score $\geqslant 1$ ) were divided into three subgroups of 'high' (activity score $\geqslant 1.75$ ), 'medium' (activity score $=1.5$ ), or 'low' (activity score $=1.0-1.25$ ). Intermediate metabolizers were defined by an activity score of $0.5-0.75$, while poor metabolizers had an activity score of 0 . This approach has been validated in a recent study correlating metoprolol PK and CYP2D6 genotype (Zineh et al, 2004).

\section{Pharmacokinetic Analyses}

Plasma concentrations of paroxetine were determined using a validated method that consists of on-line solid-phase extraction followed by liquid chromatography and mass spectroscopy. Using $0.1 \mathrm{ml}$ aliquots of plasma, this method has a lower limit of quantification (LLQ) of $0.1 \mathrm{ng} / \mathrm{ml}$. Quality and storage control (QC) plasma samples, prepared at three concentrations spanning the calibration range and stored with the experimental samples, were analyzed with each batch against separately prepared calibration standards. The results of the analysis of these QC samples were used to assess the day-to-day performance of the assay.

Paroxetine steady-state PK parameters were determined for each dosing level using the noncompartmental PK analysis computer program WinNonlin Professional, version 4.1 (Pharsight, Mountain View, CA, USA). Actual postdose sampling times were used in these calculations. The maximum observed concentration $\left(C_{\max }\right)$ and the first time of its occurrence $\left(t_{\max }\right)$ were obtained directly from the WinNonlin output, while the concentration measured at $24 \mathrm{~h}$ postdose $\left(\mathrm{C}_{(24)}\right)$ was recorded from the raw data. The area under the plasma concentration $v s$ time curve during a dosing interval $\left(\mathrm{AUC}_{(0-24)}\right)$ and the mean residence time (MRT) were calculated using a combined linear-logarithmic trapezoidal method. The apparent oral clearance $(\mathrm{CL} / \mathrm{F})$ was calculated as the quotient of dose/AUC $(0-24)$. The apparent steady-state volume of distribution $\left(V_{s s} / \mathrm{F}\right)$ following oral dosing was calculated as oral clearance multiplied by MRT. Data were summarized using descriptive statistics and were reported as the geometric mean and between-patient coefficient of variation (CV).

To calculate some (ie $4 \%$ ) of the $\mathrm{AUC}_{(0-24)}$ values, a nominal concentration of $0.05 \mathrm{ng} / \mathrm{ml}$ (ie half the LLQ) was substituted in place of those plasma concentrations below the LLQ in order to reduce downward bias in AUC estimates (Muir and Gomeni, 2004). In a few cases (ie in 2\% of the dosing occasions), a missing value at $0 \mathrm{~h}$ was substituted by the concentration at $24 \mathrm{~h}$ or vice versa.

Steady-state $C_{\max }, T_{\max }, C_{(24)}, \mathrm{AUC}_{(0-24)}, V_{\mathrm{dss}} / \mathrm{F}$, and CL/F (both before and after normalization for body weight) at each dose level were pooled across all centers and both indications, summarized by descriptive statistics, and displayed graphically to show their relationship with dose. Relationships with dose, age, weight, sex, and CYP2D6 activity scores were explored graphically. If inspection of each steady-state plasma concentration $v s$ time curve led to concerns about the validity of certain data, they were not included in the PK analyses.
A linear fixed effect model was used to compare PK parameters between groups. PK parameters were weightnormalized and log-transformed (base e) prior to the analysis. The model included a random term for patient, terms of the covariates 'weight' and 'age', and a fixed term for dose. Where both the main fixed effect and covariate term were found to be statistically significant, a term for their interaction was added to the model. All statistical tests were two-tailed, with $p<0.05$ as the accepted level for statistical significance.

As age, weight, dose, and CYP2D6 activity score may all influence the $\mathrm{PK}$ of paroxetine observed in the study population, a stepwise regression analysis was used to assess the extent to which these variables could be considered as explanatory factors for the interindividual variability in paroxetine clearance and volume of distribution.

This procedure inserts variables into an explanatory model in turn until the inclusion criteria are satisfied. At every stage of the regression, the contribution of the variables incorporated into the model in the previous stages is re-examined. A variable which may have been the best single variable to enter at an early stage may, at a later stage, be superfluous because of the relationships between it and other variables now in the regression. The partial $\mathrm{F}$ criterion for each variable in the regression at any stage of calculation is evaluated and compared with a preselected percentage point of the appropriate $\mathrm{F}$ distribution. This provides an evaluation of the contribution of each variable, irrespective of its actual point of entry into the model. Any variable that provides a nonsignificant contribution is removed from the model. This process is continued until no more variables are admitted to, or rejected from, the equation. This analysis was performed using the SAS software (Version 8.02, SAS Institute, Cary, NC). In accordance to the allometric scaling principles (West et al, 1997), a power low model was assumed to associate apparent clearance and volume of distribution parameters with weight and age. Log-log data transformation was applied to the data prior to proceeding to the stepwise regression analysis.

\section{Safety Analysis}

Patients who received at least 1 dose of study medication were included in the assessments of the safety and tolerability of paroxetine. Height, weight, blood pressure, pulse rate, 12-lead ECG, and clinical laboratory data were reviewed and summarized on an ongoing basis during the study to evaluate the safety of patients. Any clinically relevant abnormalities or values of potential clinical concern were described. All safety data were presented in tabular and/or graphical format and summarized descriptively.

\section{RESULTS}

A sample of 62 patients, including 27 children and 35 adolescents (40 males and 22 females), entered the study and received study medication. Of the children, 21 (77.8\%) had a primary diagnosis of MDD and six $(22.2 \%)$ had a primary diagnosis of OCD. For the adolescents, 31 (88.6\%) had a primary diagnosis of MDD and four (11.4\%) had a primary diagnosis of OCD. Patient summary demographics are provided in Table 1. 
Table I Demographic Characteristics of Enrolled Pediatric Patients

\begin{tabular}{|c|c|c|c|}
\hline Characteristic & $\begin{array}{l}\text { Children } \\
(n=27)\end{array}$ & $\begin{array}{l}\text { Adolescents } \\
\quad(n=35)\end{array}$ & $\begin{array}{l}\text { All patients } \\
(n=62)\end{array}$ \\
\hline \multicolumn{4}{|l|}{ Age (years) } \\
\hline Mean (SD) & $10(1.1)$ & $14(1.8)$ & $12(2.8)$ \\
\hline Range & $8-11$ & $12-17$ & $8-17$ \\
\hline \multicolumn{4}{|l|}{ Height (cm) } \\
\hline Mean (SD) & | $42.9(9.6)$ & $164.5(12.4)$ & |55.| (I5.5) \\
\hline Range & $125.5-164.0$ & |29.0-190.5 & | 25.5-190.5 \\
\hline \multicolumn{4}{|l|}{ Weight (kg) } \\
\hline Mean (SD) & 42.1 (I3.6) & $68.2(23.0)$ & $56.8(23.3)$ \\
\hline Range & $25.9-76.5$ & $30.1-14 \mid .0$ & $25.9-|4| .0$ \\
\hline Male & $74 \%$ & $57 \%$ & $65 \%$ \\
\hline \multicolumn{4}{|l|}{ Ethnic origin } \\
\hline Caucasian & $85 \%$ & $83 \%$ & $84 \%$ \\
\hline African American & $7 \%$ & $11 \%$ & $10 \%$ \\
\hline Other & $7 \%$ & $6 \%$ & $6 \%$ \\
\hline
\end{tabular}

Of the 27 children, 19 (70.4\%) completed the study, whereas eight $(29.6 \%)$ were withdrawn from the trial after receiving at least one dose of paroxetine. Reasons for discontinuation included protocol deviation $(n=3), \mathrm{AE}$ $(n=2)$, lost to followup $(n=2)$, and others (ie patient refused medication taper; $n=1)$. Of the 35 adolescents, 22 $(62.9 \%)$ completed the protocol, and 13 (37.1\%) were withdrawn prior to completing the study. Four of the adolescents withdrew due to protocol deviations, four withdrew due to an $\mathrm{AE}$, two were lost to follow up, and three were discontinued for other reasons (ie one withdrew consent, one refused medication taper and chose to be maintained on commercially available product, and one was withdrawn after day $42 \mathrm{PK}$ assessments could not be performed because of administrative reasons).

\section{Pharmacokinetic Parameters}

Of the 62 patients entered, 59 (25 children and 34 adolescents) completed the first period of dosing $(10 \mathrm{mg} /$ day) with paroxetine steady-state PK data. Fifty-five patients (24 children and 31 adolescents) completed the second period ( $20 \mathrm{mg} /$ day) with $\mathrm{PK}$ data, and 51 patients (23 children and 28 adolescents) went on to provide PK data at all three dose levels $(10,20$, and $30 \mathrm{mg} /$ day $)$. The complete data sets from two patients (one child and one adolescent) were excluded from the analysis due to internal data inconsistencies, and the data at $20 \mathrm{mg} /$ day from another adolescent patient were excluded from analysis due to a dosing error on the day prior to this assessment. In addition, examination of the plasma concentration $v s$ time curves for the 10 and $30 \mathrm{mg} /$ day doses for another child did not suggest a true steady state; thus, data from both dose
Table 2 CYP2D6 Metabolizer Status of Pediatric Patients as Assessed by CYP2D6 Genotype

\begin{tabular}{lccc}
\hline $\begin{array}{l}\text { CYP2D6 } \\
\text { metabolizer }\end{array}$ & $\begin{array}{c}\text { Children } \\
(\mathbf{n}=\mathbf{2 1})\end{array}$ & $\begin{array}{c}\text { Adolescents } \\
(\mathbf{n}=\mathbf{3 2})\end{array}$ & $\begin{array}{c}\text { All patients } \\
(\mathbf{n}=\mathbf{5 3})\end{array}$ \\
\hline Poor & 0 & 3 & 3 \\
Intermediate & 3 & 0 & 3 \\
Extensive & & & \\
$\quad$ Low & 8 & 8 & 16 \\
Medium & 3 & 7 & 10 \\
High & 7 & 14 & 21 \\
\hline
\end{tabular}

levels were deemed inevaluable. Failure to reach steady state in this patient appeared to be the result of poor adherence to dosing at both dose levels. At $10 \mathrm{mg}$, the predose concentration was four-fold lower than the corresponding value measured $24 \mathrm{~h}$ later, probably because no dose had been taken on the previous day. In addition, at $30 \mathrm{mg}$, visit assessments of adherence revealed that several doses had been missed during the previous week.

CYP2D6 genotype analysis was performed for 53 of the 59 patients providing PK data, enabling their baseline phenotypes to be predicted. As expected, the extensive metabolizer genotype (either homozygous or heterozygous for at least one functional allele) predominated. The incidence of poor metabolizers was $5.7 \%$ in this pediatric sample as a whole. No poor metabolizers were identified among the younger group, but three of the adolescents were predicted to possess this phenotype. Although one of these adolescent poor metabolizers had the highest steady-state $C_{\max }$ and $\mathrm{AUC}_{(0-24)}$ and the lowest clearance in this age-group, steady-state values in the other two poor metabolizers were less readily distinguishable from the extensive metabolizers. The distribution of poor, intermediate, and extensive metabolizers according to the activity score is presented in Table 2.

Mean steady-state paroxetine plasma concentrations over a $24-\mathrm{h}$ period, by dose and age group, are compared in Figure 1. Although the between-patient variability was considerable, mean concentrations at all three dose levels tended to be higher in the younger age group. A disproportionate increase in plasma concentrations with increasing dose was evident in both groups.

Steady-state PK parameters are summarized by dose and age group in Table 3 . The $C_{\max }$ and $\mathrm{AUC}_{(0-24)}$ data confirm that, at each dose level, paroxetine steady-state systemic exposure was higher in children (7-11 years) than in adolescents (12-17 years). However, for both parameters, the difference between age groups diminished with each increasing dose. Geometric mean values in children were approximately $100 \%$ higher than in adolescents at $10 \mathrm{mg} /$ day, but less than $30 \%$ higher at $30 \mathrm{mg} /$ day. Mean $C_{\max }$ and $\mathrm{AUC}_{(0-24)}$ values increased disproportionately with dose in both groups, but these increases were accompanied by a marked reduction in interindividual variability, as measured by CV, most notably between the 10 and $20 \mathrm{mg} /$ day dose levels. At corresponding doses, median $T_{\max }$ values in both age groups were similar. No differences due 

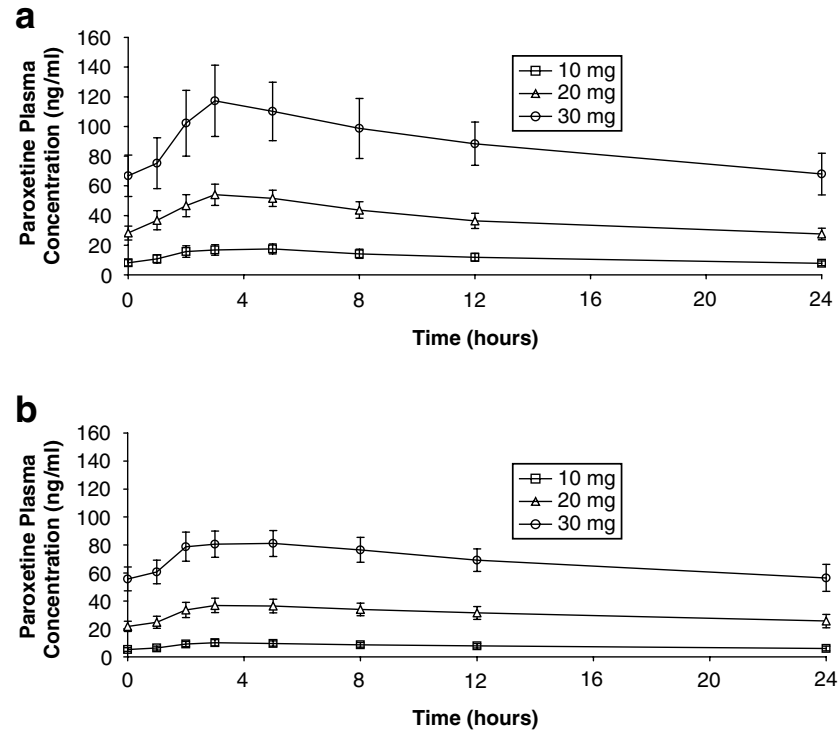

Figure I Mean ( $\pm \mathrm{SE})$ steady-state paroxetine plasma concentrations $(\mathrm{ng} / \mathrm{ml})$ over $24 \mathrm{~h}$ following once daily dosing of 10,20 , and $30 \mathrm{mg}$ in (a) children and (b) adolescents.

to sex were evident in the $C_{\max }, \mathrm{AUC}_{(0-24)}, V_{\mathrm{dss}} / \mathrm{F}$, or CL/F data in either age group at any of the three dose levels.

The geometric mean CL/F (un-normalized) at $10 \mathrm{mg} /$ day in children was approximately $50 \%$ lower than in adolescents, but only $25 \%$ lower at $20 \mathrm{mg} /$ day and $20 \%$ lower at $30 \mathrm{mg} /$ day (Figure 2a). Within each group, geometric mean $\mathrm{CL} / \mathrm{F}$ fell more than two-fold between 10 and $20 \mathrm{mg} /$ day, but by less than $40 \%$ between 20 and $30 \mathrm{mg} /$ day. As suggested by the groupwise (mean) data, $C_{\max }$ and $\mathrm{AUC}_{(0-24)}$ tended to fall with increasing age, while $\mathrm{CL} / \mathrm{F}$ and $V_{\mathrm{dss}} / \mathrm{F}$ tended to rise. Variability was, however, considerable. Mirroring the effect of age, $\mathrm{AUC}_{(0-24)}$ and $C_{\max }$ also tended to fall with increasing weight, while $\mathrm{CL} / \mathrm{F}$ and $V_{\mathrm{dss}} / \mathrm{F}$ again tended to rise. However, weight-normalized $\mathrm{CL} / \mathrm{F}$ values at each dose level appeared to remain relatively constant across the entire age range studied (8-17 years - Figure $2 \mathrm{~b}$ ), as did $V_{\mathrm{dss}} / \mathrm{F}$ values.

Stepwise regression analysis indicated that $\mathrm{CL} / \mathrm{F}$ was highly dependent on paroxetine dose, CYP2D6 activity score, and weight $(p<0.0001)$, but no significant association was found with age and sex.

The relationship between paroxetine weight-normalized CL/F, CYP2D6 status, and daily dose in pediatric patients is reported in Figure 3.

Similarly, $V_{\mathrm{dss}} / \mathrm{F}$ was strongly associated with dose, weight, and CYP2D6 activity score $(p<0.0001)$, but not significantly correlated with sex and age. These results are consistent with saturation of both clearance and first-pass effect with increasing doses.

\section{Safety Data}

Of the 62 patients entering the study, six (two children and four adolescents) withdrew due to AEs. Three of the six withdrawals occurred during the first 2 weeks of dosing at $10 \mathrm{mg} /$ day. The AEs that led to discontinuation in the adolescents were: rash in one patient on $10 \mathrm{mg} /$ day, dizziness and hyperkinesias in another patient on $20 \mathrm{mg} /$ day, hyperkinesias and mania in a third receiving $30 \mathrm{mg} /$ day, and an overdose while receiving $10 \mathrm{mg} /$ day in the fourth. The last patient had been nonadherent for several days and had taken $50 \mathrm{mg}$ on a single day to try to compensate for the missed doses. An asthma exacerbation that required treatment with prohibited medications led to study discontinuation in one child. It should be noted that the other child who discontinued from the study developed severe mania and suicidal ideation requiring hospitalization after having completed the 6-week trial, during the first week of dose tapering $(20 \mathrm{mg} /$ day $)$. The severe mania was considered by the investigator to be possibly related to study medication, and the suicidal ideation was judged to be symptomatic of the manic episode. There were two serious AEs reported during the study, which included the child with mania and suicidal ideation and the adolescent with the medication overdose described above. In contrast, most of the treatment-emergent side effects during the trial were generally mild or moderate in severity and did not lead to study discontinuation.

In two of the six cases of study discontinuation due to AEs (ie child with medication overdose; adolescent with asthma exacerbation), no PK data were available. For the remaining four cases, paroxetine plasma concentrations at the dose closest to the AEs leading to discontinuation were inspected to evaluate a potential relationship. Exposure to paroxetine $\left(C_{\max }\right.$ and $\left.\mathrm{AUC}_{(0-24)}\right)$ proximate to the $\mathrm{AE}$ was found to be in line with the average measured in the corresponding dose and age groups, with the exception of the patient withdrawn because of a rash, who had low exposure with respect to the average levels measured in the corresponding dose and age group. With regard to the CYP2D6 metabolizer status of patients with AEs leading to discontinuation, genotyping results were only available for half of the cases (ie three adolescents with rash, dizziness and hyperkinesias, and hyperkinesias and mania). None of these patients were determined to be poor metabolizers.

AEs and other safety data were enumerated on the basis of 'subject sessions', with each dose level being considered as a discrete session. Hence, each patient could have provided data from up to five subject sessions (ie 10, 20, and $30 \mathrm{mg} /$ day during the dose-rising phase, and 20 and $10 \mathrm{mg} /$ day during the tapering phase). Across the entire study sample (62 patients), there were a total of 216 subject sessions.

Overall, at least one AE was reported in 114 of the 216 subject sessions $(52.8 \%)$, with similar percentages in each age group (49.5\% for children and $55.5 \%$ for adolescents). AEs rated severe in intensity were reported in only $2.8 \%$ of sessions, again with similar percentages in each age group (3.1\% for children and $2.5 \%$ for adolescents). The most frequently reported AEs ( $\geqslant 5 \%$ of subject sessions for either age group) are listed in Table 4. Across the total study sample, headache was the most commonly reported AE (18.5\% of subject sessions), regardless of severity or age group. Abdominal pain and somnolence were the next most commonly reported AEs, although at a lower incidence (7.4 and $6.0 \%$ of subject sessions, respectively). No other $\mathrm{AE}$ exceeded an incidence of $5 \%$ in the total sample. Certain gastrointestinal AEs (diarrhea and nausea) and psychiatric AEs (aggressive reaction and nervousness) were reported 
Table 3 Summary of Paroxetine Steady-State PK Parameters in Pediatric Patients

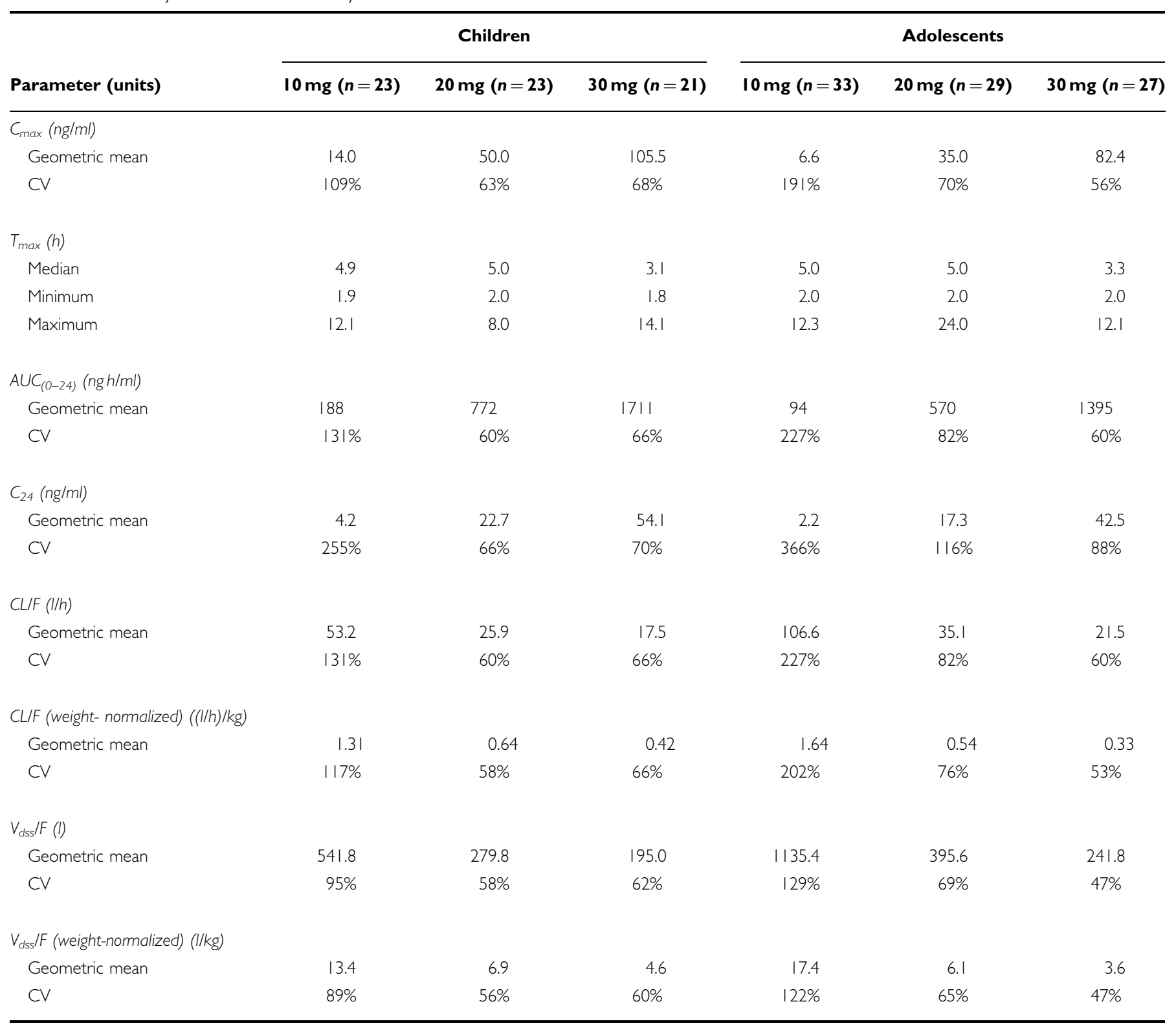

$\mathrm{CV}=$ inter individual coefficient of variation.

more frequently ( $\geqslant 2$ times the incidence) in children compared to adolescents. None of the AEs of aggressive reaction led to early withdrawal from the study; they were reported to be mild to moderate in severity and did not appear to be related to paroxetine dose (in children: two events at $10 \mathrm{mg} / \mathrm{day}$, three events at $20 \mathrm{mg} / \mathrm{day}$, and one event at $30 \mathrm{mg} / \mathrm{day}$; in adolescents: one event at $20 \mathrm{mg} /$ day).

Within both age groups, total AE frequency decreased with increasing dose from 10 to $30 \mathrm{mg} /$ day (and into the taper phase of the study). For the common AEs (headache and abdominal pain), the incidence was highest at $10 \mathrm{mg} /$ day and tended to decrease with increasing dose, in the total study population and in each age group individually. The less frequently reported AEs did not show any clear trends with dose.
No clinically significant changes in blood pressure, pulse, or ECG parameters occurred during the course of this trial. In addition, there were no apparent changes in weight in either age group. Only one patient had a value on a laboratory test that was considered to be clinically significant. At the final visit of the dose-rising stage (day 42), while on $30 \mathrm{mg} /$ day of paroxetine, this patient had an asymptomatic increase in AST to $97 \mathrm{IU} / 1$ (reference range 0-42 IU/1). At a follow-up visit 14 days later, the patient's AST had returned to the normal range (33 IU/1) while being maintained on $30 \mathrm{mg} /$ day of commercial Paxil ${ }^{\circledR}$. The patient had refused dose tapering and was discontinued from the trial at day 42 . This patient's AST at the screening and baseline visits, prior to dosing, were 23 and $20 \mathrm{IU} / \mathrm{l}$, respectively. In the opinion of the investigator, the increase in AST was considered probably unrelated to study medication. 

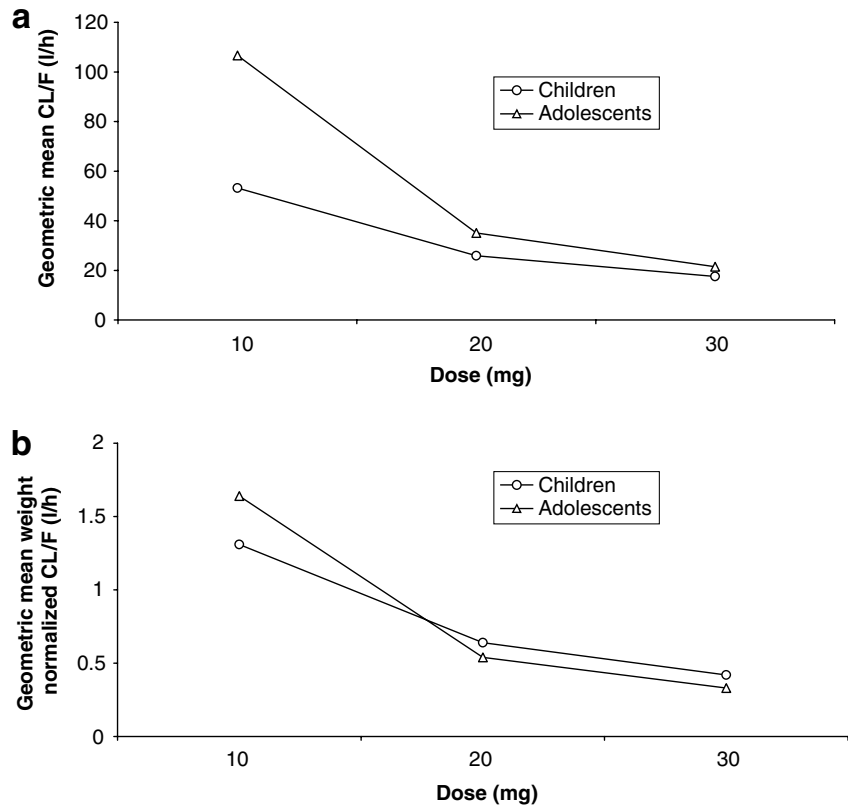

Figure 2 Relationship of (a) paroxetine oral clearance and (b) weightnormalized oral clearance to daily dose in pediatric patients.

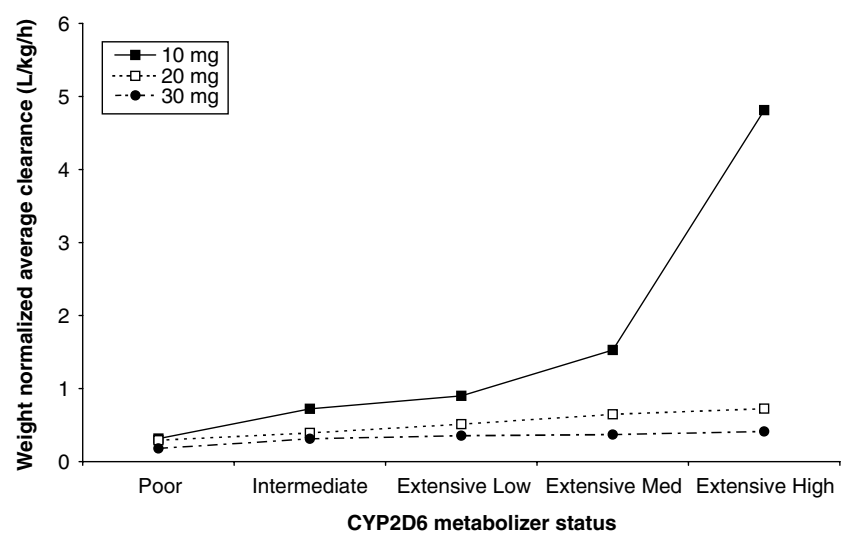

Figure 3 Relationship among paroxetine weight-normalized oral clearance, CYP2D6 metabolizer status, and daily dose in pediatric patients.

Table 4 Most Frequent Treatment-Emergent AEs by Age Group (Children and Adolescents)

\begin{tabular}{lccc}
\hline Adverse event & $\begin{array}{c}\text { Children } \boldsymbol{n} \\
(\%)\end{array}$ & $\begin{array}{c}\text { Adolescents } \\
\boldsymbol{n}(\%)\end{array}$ & $\begin{array}{c}\text { Total } \boldsymbol{n} \\
\text { (\%) }\end{array}$ \\
\hline Total subject sessions & $97(100)$ & $119(100)$ & $216(100)$ \\
Subject sessions with $\geqslant 1$ & $48(49.5)$ & $66(55.5)$ & $114(52.8)$ \\
AE & $14(14.4)$ & $26(21.8)$ & $40(18.5)$ \\
Headache & $7(7.2)$ & $9(7.6)$ & $16(7.4)$ \\
Abdominal pain & $4(4.1)$ & $9(7.6)$ & $13(6.0)$ \\
Somnolence & $7(7.2)$ & $1(0.8)$ & $8(3.7)$ \\
Diarrhea & $5(5.2)$ & $3(2.5)$ & $8(3.7)$ \\
Nausea & $6(6.2)$ & $1(0.8)$ & $7(3.2)$ \\
Aggressive reaction & $5(5.2)$ & $2(1.7)$ & $7(3.2)$ \\
Nervousness & & & \\
\hline
\end{tabular}

\section{DISCUSSION}

The present study was conducted to characterize the steadystate PK of paroxetine in children and adolescents receiving sequentially ascending doses of 10,20 , and $30 \mathrm{mg}$ once daily for successive 2-week periods. The relationships between paroxetine daily dose and steady-state pharmacokinetic parameters in the pediatric population were complex, but in all important respects mirrored those seen in adults. As in prior studies with adults (Kaye et al, 1989; Lund et al, 1979; Sindrup et al, 1992a) and the previously reported study in depressed children and adolescents (Findling et al, 1999), a nonlinear relationship between paroxetine daily dose and steady-state PK parameters was evident in this pediatric sample. Thus, both in children and adolescents, $C_{\max }$ and $\mathrm{AUC}_{(0-24)}$ increased disproportionately with dose, but this was accompanied by a marked reduction in between-patient variability.

These results are best explained in terms of CYP2D6 saturability, genetic polymorphism, ability of paroxetine to inhibit CYP2D6 activity, and the effects of these factors on oral clearance and apparent volume of distribution, both of which decreased with dose. A paroxetine doseescalation study in adults concluded that paroxetine was eliminated by two parallel pathways. The high affinity-low capacity process corresponded to CYP2D6-mediated biotransformation (Sindrup et al, 1992b). The lower affinity process(es) was not investigated by Sindrup et al (1992b), nor has it been subject to detailed characterization by others. Nonlinearity was more prominent in CYP2D6 extensive metabolizer patients, since the saturable, highaffinity process was not present in poor metabolizers (Sindrup et al, 1992b). In the current pediatric patients, the largest decreases in paroxetine clearance observed with ascending dose were seen in those patients with the greatest clearance at the initial dose level $(10 \mathrm{mg} /$ day $)$. These are the patients who are predicted to have the greatest CYP2D6 activity based on CYP2D6 genotype and, by extension, activity score. In fact, the pronounced increase in paroxetine oral clearance associated with increasing CYP2D6 activity score (Figure 3 ) is indicative of the quantitative importance of CYP2D6 activity as a determinant of paroxetine clearance, consistent with adult data (Sindrup et al, 1992a,b). However, paroxetine has also been demonstrated to inhibit CYP2D6 activity both in vitro (Crewe et al, 1992) and in vivo (Sindrup et al, 1992a). More recent data from in vitro studies indicate that paroxetine itself may inhibit CYP2D6 activity through metabolic activation of the drug by CYP2D6. The data support a mechanism involving oxidation of the methylenedioxy substitutent of paroxetine within the CYP2D6 active site to form a reactive intermediate consistent with the properties of a carbene or catechol intermediate. Either the formation of the carbene or catechol intermediate can lead to irreversible modification of the CYP2D6 protein, targeting either the iron center of the heme moiety (carbene) or nucleophilic groups of amino acids in, or near, the active site of CYP2D6. Thus, the generation of paroxetine reactive metabolites at the CYP2D6 active site subsequently leads to a loss of CYP2D6 catalytic activity (Bertelsen et al, 2003). As a consequence, as the paroxetine dose is increased, the concentration of CYP2D6 
inhibitor would also be expected to increase (Figure 1), producing a progressive increase in the extent of inhibition and thus, decrease in enzyme activity. The functional consequence of inhibition at increasing paroxetine doses would be most pronounced in those patients with the highest paroxetine clearance, since (1) higher CYP2D6 activity would produce more heme-reactive metabolite within the active site and (2) total paroxetine clearance is more dependent on CYP2D6 activity in extensive metabolizers when compared to patients classified as poor metabolizers or intermediate metabolizers. The observation that the reduction in clearance and apparent volume was generally greater during the first-dose escalation stage $(10-20 \mathrm{mg} /$ day) than during the second $(20-30 \mathrm{mg} /$ day $)$ is also consistent with this scenario.

Variability in CYP2D6 activity is generally considered to be an important determinant of the severity of dosedependent toxicity given its relationship to drug clearance. However, pharmacodynamic factors are also important. SSRIs modulate serontonergic transmission and, therefore, genetic variation in serotonin receptors could conceivably also contribute to individual susceptibility to AEs. A study of antidepressant intolerance in elderly ( $>65$ years of age) depressed outpatients found no effect of CYP2D6 genotype on the severity of AEs or the frequency of drug discontinuation. On the other hand, discontinuation of paroxetine due to side effects occurred in $46.3 \%$ of patients homozygous for $\mathrm{C}$ variant at position 102 of the serotonin $5-\mathrm{HT}_{2 \mathrm{~A}}$ receptor $(5 H T R 2 A 102 \mathrm{C} / \mathrm{C})$ compared to $16 \%$ of patients with $102 \mathrm{~T} / \mathrm{C}$ or $102 \mathrm{~T} / \mathrm{T}$ genotypes (Murphy et al, 2003). The degree to which serotonin receptor genotype may have contributed to the AEs observed in the current investigation is unknown, but developmental changes in receptor expression before, during, and after puberty must also be considered.

Three patients (5.7\%) among those consenting to CYP2D6 genotyping in this study were determined to be poor metabolizers, which is in accordance with the literature (Findling et al, 1999; Sindrup et al, 1992b). It is noteworthy that one of the three poor metabolizers had steady-state $C_{\max }$ and $\mathrm{AUC}_{(0-24)}$ that were highest in their age group, whereas the other two had values more consistent with those observed in extensive metabolizers. Although CYP2D6 is the predominant pathway for paroxetine biotransformation in most individuals, we cannot exclude the possibility that alternative cytochrome $P 450$ may be quantitatively important in a subset of patients, particularly in those deficient with respect to CYP2D6 activity. However, it should also be noted that differences in AUC between CYP2D6 poor metabolizers and extensive metabolizers become less pronounced at higher doses and with chronic dosing (Sawamura et al, 2004; Sindrup et al, 1992b) such that, for some of the 'extensive' metabolizers, CYP2D6 may be maximally inhibited and thus, they are functionally 'poor' metabolizers.

In the PK evaluable study sample, which consisted of more than 50 pediatric patients at all three dose levels spanning the ages of $8-17$, paroxetine steady-state systemic exposure $\left(C_{\max }\right.$ and $\left.\mathrm{AUC}_{(0-24)}\right)$ tended to be higher in the younger group. Systemic exposure and $V_{\mathrm{dss}} / \mathrm{F}$ appeared to be inversely related to both age and weight. Although oral clearance and volume of distribution increased with both age and weight, normalization for weight abolished the effect of age. This suggests that, as is the case for many drugs (Crom, 1994), including other SSRIs such as sertraline (Alderman et al, 1998) and fluoxetine (Wilens et al, 2002), weight may be the more important determinant of paroxetine $\mathrm{PK}$ in the pediatric population. No discernible effect of sex on PK parameters was evident.

The association of paroxetine plasma concentrations with dose and weight (age) in this study may at first appear to support a weight-based dosing recommendation in pediatric patients. However, normalization of clearance for weight did not significantly reduce the very broad between-subject PK variability at any dose level in this study (Table 1). Moreover, noting the similarities between the adolescent and adult exposure data, and the absence of a clear relationship of exposure to effectiveness in adult patients treated with paroxetine (Rasmussen and Brosen, 2000), a weight-based dosing recommendation in the pediatric patient population does not appear to be warranted.

Paroxetine was generally safe and well tolerated by both groups of pediatric patients in this study. Although AEs were reported during approximately half of all subject sessions, most were mild or moderate in intensity, and their overall frequency appeared to decrease with increasing dose and duration of exposure. Most patients (51 of 62) completed the dose-rising phase of the study, and less than $10 \%$ (6 of 62 ) were withdrawn due to AEs. There was no apparent relationship between paroxetine plasma levels and the occurrence of AEs leading to withdrawal from the study. The most common AEs were headache, abdominal pain, somnolence, diarrhea, nausea, aggressive reaction, and nervousness, each of which (except for aggressive reaction) has also been frequently observed in adult patients. Certain gastrointestinal AEs (diarrhea and nausea) and behavioral activation AEs (aggressive reaction and nervousness) were reported more frequently in children. In the current study, the aggressive reaction was more commonly reported among younger patients. This is consistent with other paroxetine pediatric studies (Geller et al, 2004; Wagner et al, 1998, 2004), in which hostility (including aggressiveness) was observed more frequently, as well in younger patients. There were no clinically significant or dose-related changes in vital signs (heart rate or blood pressure) or 12-lead ECG parameters during the current study.

The safety of antidepressants in children and adolescents, particularly the issue of possible treatment emergent suicidality, has been the focus of much attention from manufacturers of antidepressants, the Food and Drug Administration (FDA), as well as regulatory agencies in other countries. Review of these data by the FDA Advisory Committee led to the addition of new precautionary language to the label in April, 2004 and a finding in September, 2004 that $2-3 \%$ more patients on medication compared to placebo may experience an increase in suicidality (FDA, 2004a). As a result, the FDA has required antidepressants to carry 'black box' warnings about increased suicidal tendencies (FDA, 2004b). The European Committee for Medicinal Products for Human Use (CHMP) has determined that a statement advising against the use of 
paroxetine in children and adolescents under the age of 18 should be included as a special warning in the product labeling of paroxetine. In addition, the CHMP has initiated a review of all SSRIs and serotonin and norepinephrine reuptake inhibitors (SNRIs) regarding suicidality in children and adolescents. Paroxetine is not approved for use by children or adolescents. Patients who are started on therapy should be observed closely for clinical worsening, suicidality, or the development of dysfunctional behavior. Families and caregivers should be advised of the need for close observation and communication with the prescriber during paroxetine treatment.

\section{Clinical Implications}

The safety and steady-state PK data in this study have implications for dosing regimens in children and adolescents. A $10 \mathrm{mg} /$ day starting dose for pediatric patients, with the ability to increase the dose to $20 \mathrm{mg} /$ day after 4 weeks, has previously been suggested based on the findings of an open-label study of paroxetine in children and adolescents with MDD in which this dosing strategy was found to be safe and effective (Findling et al, 2002). A $10 \mathrm{mg}$ starting dose, followed by successive $10 \mathrm{mg}$ dose increments guided by clinical outcome, has been the dosing strategy employed in a number of recent double-blind studies in OCD (Geller et al, 2004) and social anxiety disorder (Wagner et al, 2004). Although paroxetine was generally well tolerated in both anxiety disorder studies, there was some evidence to suggest differences between children and adolescent patients. For example, the incidence of certain behavior-related AEs such as hyperkinesia and hostility was higher in children (Geller et al, 2004; Wagner et al, 2004) and the rate of withdrawals due to AEs was also greater in children than adolescents treated with paroxetine (Geller et al, 2004). The results of the current study demonstrated that, although the starting dose of $10 \mathrm{mg} /$ day was generally well tolerated by both children and adolescents, children had approximately twice the plasma concentration at $10 \mathrm{mg} /$ day compared to the adolescents. It can be hypothesized that these PK differences may have contributed to the AE profiles observed in other clinical trials. Furthermore, saturability of CYP2D6 and the ability of paroxetine to inhibit its own metabolism suggest that modest increases in dose may be accompanied by disproportionate increases in plasma concentrations. Therefore, a more conservative approach to dosing in children might be appropriate, such as initiating treatment with paroxetine at an even lower dose (eg $5 \mathrm{mg} /$ day) or using an extended titration schedule (eg $10 \mathrm{mg} /$ day for 4 weeks prior to any dose increase), as was employed in a previous open-label study (Findling et al, 2002).

It should be noted that these more conservative dosing strategies would not necessarily affect the disproportionate increases in paroxetine concentrations observed with increasing doses, as this observation is not likely to change over time. However, these more cautious approaches might reduce the need for higher doses by providing patients the opportunity to respond to lower doses of paroxetine, while reducing drug exposure and possible dose-related AEs. These dosing strategies should be formally evaluated in future randomized, placebo-controlled trials to confirm or refute their usefulness in children.

\section{Limitations of Study}

For the most part, this was an outpatient trial and drug adherence was only self-reported. It is possible that varying degrees of treatment adherence occurred and may have contributed to some of the interpatient variability in the observed steady-state PK parameters. Due to its primary PK objective, the study did not include a placebo-controlled arm for comparison. Therefore, it was not possible to compare rates of AEs, for example, with those of a control group. Nevertheless, the trends in the safety data from the current study appear consistent with previous observations in pediatric and/or adult patients treated with paroxetine. In addition, due to the brevity of the trial, the long-term safety of paroxetine in pediatric patients cannot be addressed with these data.

\section{ACKNOWLEDGEMENTS}

This study was sponsored by SmithKline Beecham. Dr Findling has received grant support for research from GlaxoSmithKline (GSK) and is a consultant and participant of the speakers' bureau for GSK. Five of the authors (GN, RG, RF, DJC, TMD) are employees of GSK and may own stock in the company. Funding for CYP2D6 genotyping and interpretation of the genotyping results was provided to Drs Gaedigk and Leeder by GSK. Dr Bartolic is an employee of i3 Research, a division of Ingenix Pharmaceutical Services, Inc. i3 Research sometimes provides services to GSK and its affiliates as well as to other pharmaceutical companies (some of which may be considered competitors of GlaxoSmithKline and its affiliates), including, but not limited to, services relating to this manuscript, and receives payments for these services.

\section{REFERENCES}

Alderman J, Wolkow R, Chung M, Johnston HF (1998). Sertraline treatment of children and adolescents with obsessive-compulsive disorder or depression: pharmacokinetics, tolerability and efficacy. J Am Acad Child Adolesc Psychiatry 37: 386-394.

American Psychiatric Association (1994). Diagnostic and Statistical Manual of Mental Disorders, 4th edn. (DSM-IV). American Psychiatric Association: Washington, DC, 886pp.

Bailey DG, Malcolm J, Arnold O, Spence JD (1998). Grapefruit juice-drug interactions. Br J Clin Pharmacol 46: 101-110.

Beique JC, de Montigny C, Blier P, Debonnel G (1998). Blockade of 5-hydroxytryptamine and noradrenaline uptake by venlafaxine: a comparative study with paroxetine and desipramine. $\mathrm{Br} J$ Pharmacol 125: 526-532.

Bertelsen KM, Venkatakrishnan K, von Moltke LL, Obach RS, Greenblatt DJ (2003). Apparent mechanism-based inhibition of human CYP2D6 in vitro by paroxetine: comparison with fluoxetine and quinidine. Drug Metab Disp 31: 289-293.

Bloomer JC, Woods FR, Haddock RE, Lennard MS, Tucker GT (1992). The role of cytochrome P4502D6 in the metabolism of paroxetine by human liver microsomes. Br J Clin Pharmacol 33: 521-523.

Braconnier A, Le Coent R, Cohen D, DEROXADO Study Group (2003). Paroxetine versus clomipramine in adolescents with 
severe major depression: a double-blind, randomized, multicenter trial. J Am Acad Child Adolesc Psychiatry 42: 22-29.

Crewe HK, Lennard MS, Tucker GT, Woods FR, Haddock RE (1992). The effect of selective serotonin re-uptake inhibitors on cytochrome P4502D6 (CYP2D6) activity in human liver microsomes. Br J Clin Pharmacol 34: 262-265.

Crom WR (1994). Pharmacokinetics in the child. Environ Health Perspect 102(Suppl 11): 111-117.

DeVane CL (1992). Pharmacokinetics of the selective serotonin reuptake inhibitors. J Clin Psychiatry 53(Suppl): 13-20.

Diler RS, Tamam L, Avci A (2000). Withdrawal symptoms associated with paroxetine discontinuation in a nine-year-old boy. J Clin Psychopharmacol 20: 586-587.

Findling RL, Myers C, O’Riordan MA, Branicky LA, Pettigrew A, Reed MD et al (2002). An open-label dosing study of paroxetine in depressed youths. Curr Ther Res 63: 588-601.

Findling RL, Reed MD, Myers C, O'Riordan MA, Fiala S, Branicky $\mathrm{L}$ et al (1999). Paroxetine pharmacokinetics in depressed children and adolescents. J Am Acad Child Adolesc Psychiatry 38: 952-959.

Food and Drug Administration (2004a). FDA Statement on Recommendations of the Psychopharmacologic Drugs and Pediatric Advisory Committees. Available at: http://www.fda. gov/bbs/topics/news/2004/NEW01116.html (accessed September 30, 2004).

Food and Drug Administration (2004b). FDA Launches a MultiPronged Strategy to Strengthen Safeguards for Children Treated With Antidepressant Medications. Available at: http://www.fda. gov/bbs/topics/news/2004/NEW01124.html (accessed October 15, 2004).

Gaedigk A, Bhathena A, Ndjountsche L, Pearce RE, Abdel-Rahman SM, Alander SW et al (2005a). Identification and characterization of novel sequence variations in the cytochrome P4502D6 (CYP2D6) gene in African Americans. Pharmacogenomics J print copy in press (originally published online March 15, 2005, at www.nature.com/tpj/ ).

Gaedigk A, Bradford LD, Marcucci KA, Leeder JS (2002). Unique CYP2D6 activity distribution and genotype-phenotype discordance in African Americans. Clin Pharmacol Ther 72: 76-89.

Gaedigk A, Gotschall RR, Forbes NS, Simon SD, Leeder JS (1999). Optimization of cytochrome P4502D6 (CYP2D6) phenotype assignment using a genotyping algorithm based on allele frequency data. Pharmacogenetics 9: 669-682.

Gaedigk A, Ndjountsche L, Bradford LD, Leeder JS (2005b). Limited association of the $2988 \mathrm{G}>\mathrm{A}$ SNP with CYP2D6*41 in African Americans. Clin Pharmacol Ther 77: 228-230.

Gaedigk A, Ndjountsche L, Gaedigk R, Bradford LD, Leeder JS (2003a). Discovery of a novel non-functional cytochrome P4502D6 allele, CYP2D6*42, in African-Americans. Clin Pharmacol Ther 73: 575-576.

Gaedigk A, Ryder DL, Bradford LD, Leeder JS (2003b). CYP2D6 poor metabolizer status can be ruled out by a single genotyping assay testing for the $-1584 \mathrm{G}$ promoter polymorphism. Clin Chem 49: 1008-1011.

Geller DA, Biederman J, Stewart SE, Mullin B, Farrell C, Wagner $\mathrm{KD}$ et al (2003). Impact of comorbidity on treatment response to paroxetine in pediatric obsessive-compulsive disorder: is the use of exclusion criteria empirically supported in randomized clinical trials? J Child Adolesc Psychopharmacol 13(Suppl 1): S19-S29.

Geller DA, Wagner KD, Emslie G, Murphy T, Carpenter DJ, Wetherhold E et al (2004). Paroxetine treatment in children and adolescents with obsessive-compulsive disorder: a randomized, multicenter, double-blind placebo-controlled trial. J Am Acad Child Adolesc Psychiatry 43: 1387-1396.

Haddock RE, Jackson D, Woods FR (1989). Paroxetine: lack of effect on hepatic drug metabolizing enzymes. Acta Psychiatr Scand 350(Suppl): 93-94.
Kaufman J, Birmaher B, Brent D, Rao U, Flynn C, Moreci P et al (1997). Schedule for affective disorders and schizophrenia in school-age children - present and lifetime version (K-SADS-PL): initial reliability and validity data. J Am Acad Child Adolesc Psychiatry 36: 980-988.

Kaye CM, Haddock RE, Langley PF, Mellows G, Tasker TC, Zussman BD et al (1989). A review of the metabolism and pharmacokinetics of paroxetine in man. Acta Psychiatr Scand Suppl 350: 60-75.

Keller MB, Ryan ND, Strober M, Klein RG, Kutcher SP, Birmaher B et al (2001). Efficacy of paroxetine in the treatment of adolescent major depression: a randomized, controlled trial. J Am Acad Child Adolesc Psychiatry 40: 762-772.

Lund J, Lomholt B, Fabricius J, Christensen JA, Bechgaard E (1979). Paroxetine: pharmacokinetics, tolerance and depletion of blood 5-HT in man. Acta Pharmacol Toxicol (Copenh) 44: 289-295.

Marsden CA, Tyrer P, Casey P, Seivewright N (1987). Changes in human whole blood 5-hydroxytryptamine (5-HT) and platelet 5HT uptake during treatment with paroxetine, a selective 5-HT uptake inhibitor. J Psychopharmacol 1: 244-250.

Milin RP, Simeon J, Spenst WP (1999). Double-blind study of paroxetine in adolescents with unipolar depression. Poster presented at the American Academy of Child and Adolescent Psychiatry (AACAP) Annual Meeting, Chicago, IL, October 19-24, NR63, pp 104-105 (abstract).

Muir K, Gomeni R (2004). Non-compartmental analysis. In: Bonate PL, Howard DR (eds). Pharmacokinetics in Drug Development: Clinical Study Design and Analysis. AAPS Press: Arlington, VA. pp 235-266.

Murphy Jr GM, Kremer C, Rodrigues HE, Schatzberg AF (2003). Pharmacogenetics of antidepressant medication intolerance. Am J Psychiatry 160: 1830-1835.

National Institute of Mental Health (1985). Special feature: rating scales and assessment instruments for use in pediatric psychopharmacology research. Psychopharmacol Bull 21: 839-842.

Ozdemir V, Tyndale RF, Reed K, Herrmann N, Sellers EM, Kalow $\mathrm{W}$ et al (1999). Paroxetine steady-state plasma concentration in relation to CYP2D6 genotype in extensive metabolizers. J Clin Psychopharmacol 19: 472-475.

Poznanski EO, Freeman LN, Mokros HB (1985). Children's depression rating scale revised. Psychopharmacol Bull 21: 979-989.

Raptopoulos P, McClelland GR, Jackson D (1989). The clinical pharmacology of paroxetine in healthy subjects. Acta Psychiatr Scand Suppl 350: 46-48.

Rasmussen BB, Brosen K (2000). Is therapeutic drug monitoring a case for optimizing clinical outcome and avoiding interactions of the selective serotonin reuptake inhibitors? Ther Drug Monit 22: $143-154$.

Rey-Sanchez F, Gutierrez-Casares JR (1997). Paroxetine in children with major depressive disorder: an open trial. J Am Acad Child Adolesc Psychiatry 36: 1443-1447.

Sawamura K, Suzuki Y, Someya T (2004). Effects of dosage and CYP2D6-mutated allele on plasma concentration of paroxetine. Eur J Clin Pharmacol 60: 553-557.

Scahill L, Riddle MA, McSwiggin-Hardin M, Ort SI, King RA, Goodman WK et al (1997). Children's Yale-Brown obsessive compulsive scale: reliability and validity. J Am Acad Child Adolesc Psychiatry 36: 844-852.

Sindrup SH, Brosen K, Gram LF (1992a). Pharmacokinetics of the selective serotonin reuptake inhibitor paroxetine: nonlinearity and relation to the sparteine oxidation polymorphism. Clin Pharmacol Ther 51: 288-295.

Sindrup SH, Brosen K, Gram LF, Hallas J, Skjelbo E, Allen A et al (1992b). The relationship between paroxetine and the sparteine oxidation polymorphism. Clin Pharmacol Ther 51: 278-287. 
Thomas DR, Nelson DR, Johnson AM (1987). Biochemical effects of the antidepressant paroxetine, a specific 5-hydroxytryptamine uptake inhibitor. Psychopharmacology (Berl) 93: 193-200.

Tulloch IF, Johnson AM (1992). The pharmacologic profile of paroxetine, a new selective serotonin reuptake inhibitor. J Clin Psychiatry 53(Suppl): 7-12.

Wagner KD, Berard R, Stein MB, Wetherhold E, Carpenter DJ, Perera $\mathrm{P}$ et al (2004). A multicenter, randomized, double-blind, placebo-controlled trial of paroxetine in children and adolescents with social anxiety disorder. Arch Gen Psychiatry 61: $1153-1162$.

Wagner KD, Birmaher B, Carlson G, Clarke G, Emslie G, Geller B et al (1998). Safety of paroxetine and imipramine in the treatment of adolescent depression. Presented at the Annual
Meeting of the NIMH New Clinical Drug Evaluation Unit (NCDEU), Boca Raton, FL, USA, 10-13 June 1998.

Wagstaff AJ, Cheer SM, Matheson AJ, Ormrod D, Goa KL (2002). Spotlight on paroxetine in psychiatric disorders in adults. CNS Drugs 16: 425-434.

West GB, Brown JH, Enquist BJ (1997). A general model for the origin of allometric scaling laws in biology. Science 276: 122-126. Wilens TE, Cohen L, Biederman J, Abrams A, Neft D, Faird N et al (2002). Fluoxetine pharmacokinetics in pediatric patients. J Clin Psychopharmacol 22: 568-575.

Zineh I, Beitelshees AL, Gaedigk A, Walker JR, Pauly DF, Eberst K et al (2004). Pharmacokinetics and CYP2D6 genotypes do not predict metoprolol adverse events or efficacy in hypertension. Clin Pharmacol Ther 76: 536-544. 